\title{
Avaliação da qualidade óptica de lentes de contato gelatinosas na correção de miopia
}

\author{
Evaluation of optical performance of soft contact lenses in myopic correction
}

\author{
Marcelo Weslley Dalcoll ${ }^{1}$ \\ Mílton Ruiz Alves ${ }^{2}$ \\ Jackson Barreto Jr. ${ }^{3}$ \\ Iris de Souza Yamane ${ }^{4}$ \\ Samir Bechara ${ }^{5}$ \\ Adriana Mukai ${ }^{6}$
}

Trabalho realizado na Faculdade de Medicina da Universidade de São Paulo - USP - São Paulo (SP) - Brasil.

${ }^{1}$ Estagiário da Clínica Oftalmológica do Hospital das Clínicas da Faculdade de Medicina da Universidade de São Paulo - USP - São Paulo (SP) - Brasil.

2 Professor Livre Docente pela USP - São Paulo (SP) Brasil.

${ }^{3}$ Pós-graduando pela Faculdade de Medicina da USP São Paulo (SP) - Brasil.

${ }^{4}$ Estagiária da Clínica Oftalmológica do Hospital das Clínicas da Faculdade de Medicina da USP - São Paulo (SP) - Brasil.

${ }^{5}$ Professor Livre Docente pela USP - São Paulo (SP) Brasil.

${ }^{6}$ Técnica Oftálmica do Serviço de Refrativa da Clínica Oftalmológica do Hospital das Clínicas da Faculdade de Medicina da USP - São Paulo (SP) - Brasil.

Endereço para correspondência: Milton Ruiz Alves. Rua Capote Valente, 432 - Cj. 155 - São Paulol (SP) CEP 05409-001

E-mail: milton.r.alves@uol.com.br

Os autores não possuem interesse financeiro no desenvolvimento ou marketing das medicações ou instrumentos referidos no estudo.

\section{RESUMO}

Objetivo: Avaliar a qualidade óptica de olhos adaptados com dois tipos diferentes de lentes de contato gelatinosas: Acuvue ${ }^{\circledR} 2$ (Vistacon J\&J Vision Care Inc., EUA) e World Vision Disposable Asférica Wave Front ${ }^{\mathbb{B}}$ (World Vision Ophthalmic, Brasil). Métodos: Estudo clínico e prospectivo com intervenção em amostra constituída por 40 indivíduos portadores de miopia entre $-0,75 \mathrm{D}$ e $-4,50 \mathrm{D}$, sem ou com astigmatismo até $-0,75 \mathrm{D}$. Um olho de cada paciente foi sorteado para avaliação a qual incluiu acuidade visual de alto e baixo contraste, aberrometria e teste de sensibilidade ao contraste. Resultados: A sobre-refração objetiva realizada pelo Nidek OPD-Scan detectou hipercorreção significativa para ambas as lentes de contato gelatinosas. A avaliação da AV $\log$ MAR de alto e baixo contraste mostrou diferença significativa favorável das lentes de contato gelatinosas em relação aos óculos e na comparação entre as lentes de contato gelatinosas, apenas em relação à AV $\log \mathrm{MAR}$ de alto contraste, houve melhor desempenho da LC World Vision Disposable Asférica Wave Front ${ }^{\circledR}$. A avaliação de sensibilidade ao contraste detectou melhor desempenho das lentes de contato gelatinosas em relação aos óculos para as freqüências espaciais de 1,5; 3; 6 e 18. Não houve diferença significativa entre os dois tipos de lentes de contato gelatinosas nas freqüências espaciais avaliadas. A análise de frentes de onda não identificou diferença significativa do índice de Strehl, função de transferência modular (MTF) e das aberrações de alta ordem (aberrações totais, coma, trefoil, quadrifoil, esférica e astigmatismo secundário) sem e com as lentes de contato gelatinosas, bem como na comparação entre elas. Conclusão: Houve melhor desempenho óptico da lente de contato gelatinosa World Vision Disposable Asférica Wave Front ${ }^{\circledR}$ na medida de AV logMAR de alto contraste. Não houve diferença entre as lentes de contato gelatinosas nas avaliações de acuidade visual logMAR de baixo contraste, sensibilidade ao contraste, índice de Strehl, MTF e das aberrações de alta ordem.

Descritores: Miopia; Óculos; Lentes de contato; Acuidade visual; Sensibilidades de contraste; Estudos prospectivos

\section{INTRODUÇÃO}

A qualidade óptica de olhos adaptados com lentes de contato (LC) rígidas ou gelatinosas depende não somente da óptica ocular, mas também das propriedades ópticas das LC e das suas interações com o olho, especialmente, com a córnea e filme lacrimal ${ }^{(1)}$. A qualidade óptica da LC depende do desenho e da regularidade da construção das superfícies anterior e posterior da lente, assim como da homogeneidade do índice de refração do material utilizado na sua fabricação ${ }^{(1)}$. 
A grande variedade de LC gelatinosas (LCG) disponível no mercado resulta de diferentes desenhos, materiais e métodos de fabricação. Diferenças no processo de manufatura produzem LCG com propriedades ópticas distintas ${ }^{(2-5)}$. Daí a necessidade da realização de pesquisas para avaliar o desempenho óptico dessas lentes. Este estudo teve por objetivo avaliar a qualidade óptica de olhos adaptados com dois diferentes tipos de LCG, analisada por meio de medida da sensibilidade ao contraste e de aberrometria.

\section{MÉTODOS}

Realizou-se estudo clínico prospectivo com intervenção, aprovado pelo Comitê de Ética para Análise de Projetos de Pesquisa-CAPPesq, da Diretoria Clínica do Hospital das Clínicas e da Faculdade de Medicina da Universidade de São Paulo (FMUSP). Os indivíduos incluídos na investigação receberam informações detalhadas da natureza da pesquisa e, após os esclarecimentos, assinaram o termo de consentimento livre e esclarecido.

No Setor de Doenças Externas e Córnea do Hospital das Clínicas da FMUSP, entre julho e dezembro de 2007, foram selecionados indivíduos portadores de miopia com ou sem astigmatismo, que preencheram os critérios de inclusão e exclusão do estudo. Os critérios de inclusão adotados foram os seguintes: indivíduos de ambos os sexos, portadores de miopia entre $-0,75$ e $-4,50 \mathrm{D}$, sem ou com astigmatismo de até $-0,75$ $\mathrm{D}$, não usuários de $\mathrm{LC}$, com acuidade visual (AV) corrigida com óculos igual ou superior a 20/20 e com capacidade de compreender as orientações recomendadas de uso e manuseio das LCG. Adotaram-se os seguintes critérios de exclusão: presença de afecção ocular ativa de natureza alérgica, inflamatória ou infecciosa ou indivíduos que não puderam ser adaptados com os dois tipos de LCG (Quadro). Todos os participantes foram inicialmente submetidos à anamnese, exame ocular externo, tonometria de não-contato, ceratometria, refratometria dinâmica subjetiva, medida da acuidade visual com correção, fundoscopia direta, biomicroscopia do segmento anterior e avaliação da relação LCG-córnea feita à lâmpada de fenda, solicitando-se ao paciente para olhar na direção da testa do examinador, piscar corretamente e, depois, olhar para

\begin{tabular}{|lcc|}
\hline \multicolumn{2}{|c|}{ Quadro. Características das lentes de contato gelatinosas avaliadas } \\
Acuvue ${ }^{\circledR}$ & $\begin{array}{c}\text { World Vision } \\
\text { Disposable Asférica } \\
\text { Wave Front }\end{array}$ \\
Curva base $(\mathrm{mm})$ & 8,60 & 8,70 \\
Diâmetro $(\mathrm{mm})$ & 14,0 & 14,5 \\
Conteúdo aquoso $(\%)$ & 58,0 & 55,0 \\
Espessura central $(\mathrm{mm})$ & 0,07 para -3,00 D & 0,09 para -3,00 D \\
Material & Etafilcon A & Metafilcon A \\
Curva posterior & Esférica & Asférica \\
Processo de fabricação & Moldada & Moldada \\
\hline
\end{tabular}

os lados. Considerou-se movimentação adequada da LCG um deslocamento lateral de $1 \mathrm{~mm}$ e vertical de 0,5 a $1 \mathrm{~mm}$ ao movimento ocular. A avaliação da centralização, movimentação e a sobre-refração foram executadas após vinte minutos da colocação da LCG.

A amostra do estudo foi constituída por 40 indivíduos com idades entre 18 e 43 anos (média e desvio padrão de 29,3 \pm 7,5 anos). Trinta e um (77,5\%) eram do sexo feminino e em relação aos componentes da refração, apresentavam média e desvio padrão do componente esférico de $-1,19 \pm 1,50 \mathrm{D}$; média e desvio padrão do componente cilíndrico de $-0,60 \pm 0,40 \mathrm{D}$ e média e desvio padrão do equivalente esférico de $-1,50 \pm 1,50 \mathrm{D}$. Um olho da cada paciente foi sorteado para avaliação. Os participantes, posteriormente, retornaram para a adaptação das LCG, quando foram submetidos às medidas da AV logMAR de alto e baixo contraste e às avaliações de sensibilidade ao contraste e estudo aberrométrico. A seqüência de adaptação das duas LCG foi definida previamente por sorteio. Entre cada avaliação fez-se um intervalo de 30 minutos.

A medida da AV $\log$ MAR de alto contraste foi realizada utilizando a tabela parametrizada de Bailey-Lovie a $4 \mathrm{~m}$ sob condições padronizadas de iluminação de $70-110 \mathrm{~cd} / \mathrm{m}^{2}$. Para a medida da AV logMAR de baixo contraste, utilizou-se a tabela Bailey-Lovie de baixo contraste $(10 \%)$ sob as mesmas condições de luminosidade. O teste de sensibilidade ao contraste foi realizado com o emprego da tabela "Vision Contrast Test System" (VCTS 6500, Vistech Consultants Inc), que verifica freqüências espaciais diferentes $(1,5 ; 3 ; 6 ; 12$ e 18), representadas em ciclos por graus, distribuídas em cinco fileiras (A, B, C, D e E). O indivíduo foi posicionado a 2,4 m (8 pés) da tabela, sem LCG e depois com cada uma das LCG. Cada freqüência testada iniciou-se com alto nível de contraste, o qual foi diminuído progressivamente até o círculo de menor contraste que o paciente pode enxergar. A última resposta que identificava corretamente o padrão determinou o limiar de contraste para determinada freqüência espacial testada.

A sobre-refração e as medidas do índice de Strehl, da função de transferência modular (MTF) e das aberrações ópticas de alta ordem foram obtidas com o emprego do aberrômetro Nidek OPD-Scan. Todas as medidas foram realizadas com a pupila em midríase de pelo menos $6 \mathrm{~mm}$ de diâmetro. Para a obtenção da midríase, instilou-se uma gota de fenilefrina a $10 \%$, vinte minutos antes do exame. $\mathrm{O}$ exame foi repetido três vezes. Para efeito de cálculo, foram consideradas as médias dos três exames.

Para o processamento dos dados, foi construído um banco de dados com o software Access do Office 2000. As análises estatísticas foram realizadas com o auxílio dos programas de computador SPSS 13.0, StatXact 4.0 e GraphPad InStat 3.05. Foram empregados os seguintes testes estatísticos: ANOVA bidirecional de Friedman e o teste de Wilcoxon para duas amostras pareadas não paramétricas. Os valores de $\mathrm{p}<0,05$ foram considerados estatisticamente significativos. 


\section{RESULTADOS}

A tabela 1 mostra os resultados da sobre-refração pelo aberrômetro com as duas LCG, para um diâmetro pupilar de $6 \mathrm{~mm}$.

Houve diferença significativa em relação aos componentes esfera $(p<0,01)$ e equivalente esférico $(p<0,01)$ residuais entre as duas lentes. A LC World Vision Disposable Asférica Wave Front ${ }^{\circledR}$ apresentou hipercorreção de cerca de $0,2 \mathrm{D}$ a mais que a LC Acuvue $2^{\circledR}$.

A tabela 2 mostra os resultados da avaliação da AV logMAR de alto e baixo contraste com óculos e com as LCG avaliadas.

Houve diferença significativa favorável das LCG em relação aos óculos $(\mathrm{p}=0,000)$. Na comparação entre as LCG, em relação à AV $\log \mathrm{MAR}$ de alto contraste, houve melhor desempenho da LC World Vision Disposable Asférica Wave Front ${ }^{\circledR}$ $(\mathrm{p}=0,012)$.

A figura 1 mostra os resultados da sensibilidade ao contraste com os óculos e com as LCG avaliadas. Os resultados do teste de sensibilidade ao contraste mostraram melhor desempenho das LCG em relação aos óculos para as freqüências espaciais de 1,$5 ; 3 ; 6$ e 18 . Não houve diferença significativa entre os dois tipos LCG nas freqüências espaciais avaliadas.

As figuras 2 e 3 mostram, respectivamente, os resultados obtidos para o índice de Strehl e função de transferência modular. Não houve mudanças significativas em ambas as medidas de qualidade óptica, tanto entre óculos e LCG como entre as LCG avaliadas.

As aberrações de alta ordem (terceira e quarta ordem) estão representadas na figura 4 . As médias da raiz quadrada média de cada aberração se mostraram muito similares, não houve diferença entre as medidas com óculos e LCG bem como entre os dois tipos de LCG avaliados.

\begin{tabular}{|c|c|c|}
\hline \multirow{2}{*}{$\begin{array}{l}\text { Componente } \\
\text { refrativo } \\
\text { residual }\end{array}$} & \multicolumn{2}{|c|}{$\begin{array}{l}\text { Tipo de lente de } \\
\text { contato }(n=40)\end{array}$} \\
\hline & Acuvue $2^{\circledR}$ & $\begin{array}{c}\text { World Vision Disposable } \\
\text { Asférica Wave Front }{ }^{\circledR}\end{array}$ \\
\hline Esfera & $+0,7 \pm 1,2$ & $+0,9 \pm 0,9$ \\
\hline Cilindro & $-0,6 \pm 0,4$ & $-0,6 \pm 0,6$ \\
\hline Equivalente esférico & $+0,4 \pm 1,2$ & $+0,6 \pm 0,9$ \\
\hline
\end{tabular}

\begin{tabular}{|c|c|c|}
\hline \multirow[b]{2}{*}{$\begin{array}{l}\text { Correção } \\
\text { óptica }\end{array}$} & \multicolumn{2}{|c|}{$(n=40)$} \\
\hline & $\begin{array}{l}\text { AV } \log M A R \\
\text { Alto contraste }\end{array}$ & $\begin{array}{c}\text { AV logMAR } \\
\text { Baixo contraste }\end{array}$ \\
\hline Óculos & $-0,06 \pm 0,12^{*}$ & $0,19 \pm 0,10^{*}$ \\
\hline LC Acuvue $2^{\circledR}$ & $-0,13 \pm 0,11^{*}$ & $0,10 \pm 0,10$ \\
\hline $\begin{array}{l}\text { World Vision Disposable } \\
\text { Asférica Wave Front }{ }^{\circledR}\end{array}$ & $-0,17 \pm 0,08^{*}$ & $0,07 \pm 0,14$ \\
\hline
\end{tabular}

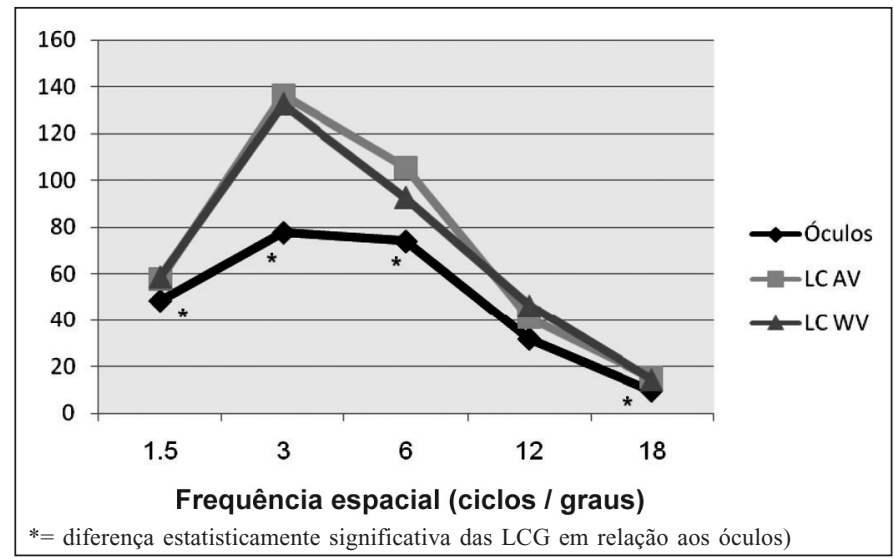

Figura 1 - Teste de sensibilidade ao contraste (em ciclos/grau) com óculos e com as lentes Acuvue $2^{\circledR}$ (AV) e World Vision Disposable Asférica Wave Front $^{\oplus}$ (WV) $(n=40)$

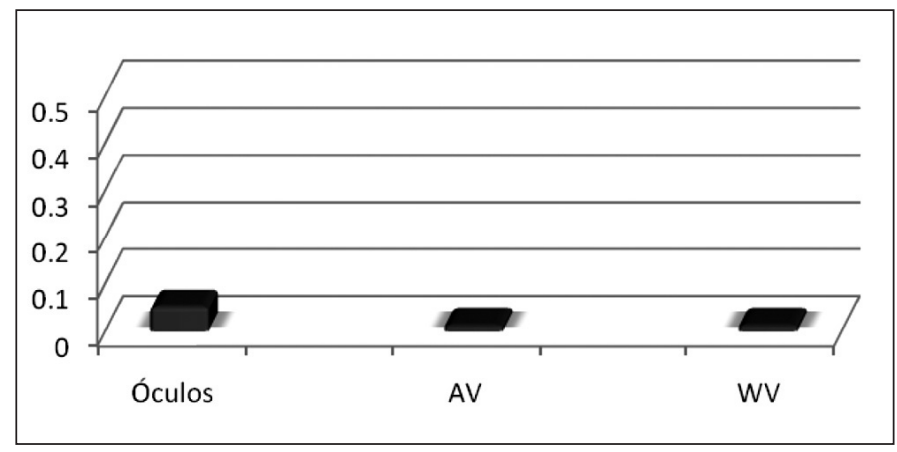

Figura 2 - Índice de Strehl com óculos e com as lentes Acuvue $2^{\circledR}$ (AV) e World Vision Disposable Asférica Wave Front ${ }^{\circledR}$ (WV) $(n=40)$

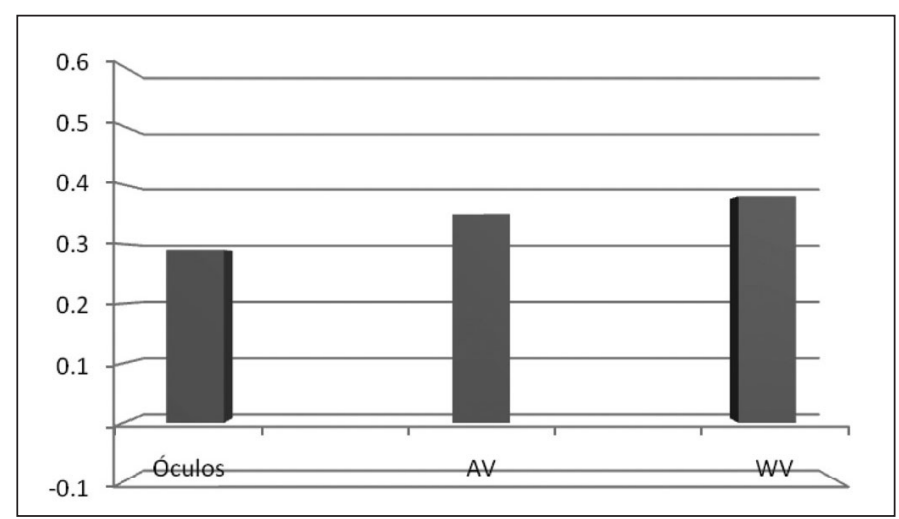

Figura 3 - Área sob a curva da função de transferência modular com óculos e com as lentes Acuvue $2^{\circledR}$ (AV) e World Vision Disposable Asférica Wave Front ${ }^{\oplus}$ (WV) $(n=40)$

\section{DISCUSSÃO}

Neste estudo, todos os participantes tiveram os seus erros refrativos corrigidos com óculos e com as duas LCG avaliadas. Todos os participantes foram submetidos a medidas repetidas de AV logMAR de alto e baixo contraste, sensibilida- 


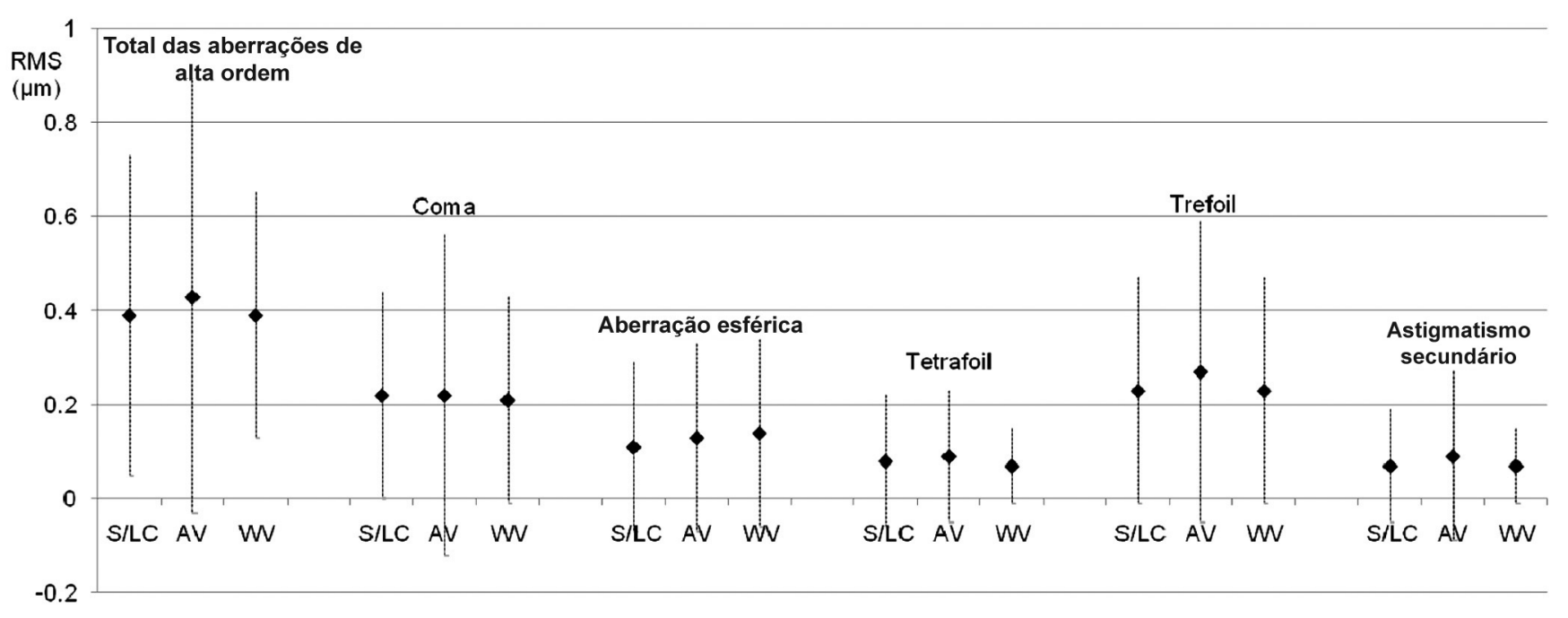

Figura 4 - Raiz quadrada média (média \pm 2 desvios padrão) do total das aberrações de alta ordem, coma, aberrações esféricas, tetrafoil, trefoil e astigmatismo secundário sem lentes de contato (S/LC) e com as lentes Acuvue $2^{\circledR}$ (AV) e World Vision Disposable Asférica Wave Front ${ }^{\circledR}($ WV) $(n=40)$

de ao contraste e de aberrações de alta ordem, evitando-se, assim, a composição de grupos. Dessa forma, buscou-se eliminar a variação interindivíduos. As duas LCG utilizadas foram selecionadas com o mesmo poder refrativo para cada olho avaliado. No entanto, os resultados da sobre-refração pelo aberrômetro Nidek OPD-Scan, para o diâmetro pupilar de $6 \mathrm{~mm}$, mostram uma diferença estatisticamente significativa de $+0,2 \mathrm{D}$ para os componentes esfera e equivalente esférico entre as lentes e maior hipercorreção relacionada à LC World Vision Disposable Asférica Wave Front ${ }^{\circledR}$ (Tabela 1). Contudo, esta diferença refrativa não é clinicamente importante visto que a AV $\log M A R$ de alto contraste foi superior para a LCG World Vision Disposable Asférica Wave Front ${ }^{\circledR}$ (Tabela 2). Adicionalmente, a amostra analisada foi composta de adultos jovens (29,3 $\pm 7,5$ anos), o que justificaria a compensação desse componente residual positivo pelo efeito acomodativo.

Quando um objeto escuro se encontra em um fundo claro (alto contraste), o fator limitante para a detecção deste objeto é o tamanho ou a distância que ele se encontra do olho humano, ou seja, o objeto tem que apresentar uma dimensão mínima para ser percebido ${ }^{(6-7)}$. Nesta investigação, quando se utilizou a medida da AV logMAR de alto contraste como avaliação de qualidade óptica visual, verificou-se desempenho superior no uso de LCG e na comparação entre as LCG, melhor desempenho no uso da LC World Vision Disposable Asférica Wave Front ${ }^{\circledR}$ (Tabela 2). Quando um objeto claro se encontra em um fundo escuro (baixo contraste), o fator limitante para a detecção do objeto não é mais a sua extensão espacial, mas, sim, a diferença de contraste gerada pela energia luminosa transmitida ${ }^{(6-7)}$. Neste estudo, considerando-se a medida da AV $\log$ MAR de baixo contraste, houve desempenho visual superior no uso de LCG e não foi detectada diferença na comparação entre as LCG. A sensibilidade ao contraste é uma medida mais sensível da função visual que a AV de alto e baixo contraste, não somente na avaliação com LCG, mas também em vários estados de doença ocular ${ }^{(5,8-9)}$. Nesta pesquisa houve diferença na comparação entre óculos e LCG exceto na freqüência espacial de $12 \mathrm{cpg}$. Não houve diferença na comparação entre as LCG nas freqüências espaciais avaliadas (Figura 1). As LCG testadas apresentam alto conteúdo aquoso e praticamente a mesma espessura central (Quadro). A redução de sensibilidade ao contraste em freqüências mais baixas foi observada em LCG com baixo conteúdo de água $^{(10)}$ e com maior espessura central ${ }^{(11)}$. A influência de LCG na $\mathrm{AV}$ e na sensibilidade ao contraste tem sido relatada nos usuários de $\mathrm{LCG}^{(12-14)}$ e o pior desempenho creditado à indução de aberrações de alta ordem pelas LCG. Neste estudo não foram identificadas diferenças sem e com LCG e mesmo na comparação entre as LCG avaliadas no que concerne às aberrações totais, coma, trefoil, quadrifoil, esférica e astigmatismo secundário (Figura 4).

O total das aberrações de alta ordem foi de $0,43 \pm 23 \mu \mathrm{m}$ para LCG Acuvue $2^{\circledR} \mathrm{e} 0,39 \pm 0,13 \mu \mathrm{m}$ para LCG World Vision Disposable Asférica Wave Front ${ }^{\circledR}$. Alguns autores ${ }^{(15)}$ relataram valores similares $(0,455 \pm 0,15 \mu \mathrm{m})$, utilizando o mesmo aberrômetro, aos encontrados para uma amostra de míopes não usuários de LCG, o que corrobora com a não indução de aberrações de alta ordem com as LCG estudadas. No entanto, estudo prévio realizado para comparar o desempenho visual de indivíduos portadores de miopia com ou sem astigmatismo, corrigidos com LCG Acuvue $2^{\circledR}$, Biomedics ${ }^{\circledR} 55$ e Focus ${ }^{\circledR}$ 1-2 week verificou uma diferença significativa na aberração de terceira ordem com e sem as LCG. Em relação ao nível trefoil de aberração de terceira ordem, o desempenho óptico das LCG Acuvue $2^{\circledR}$ e Biomedics ${ }^{\circledR} 55$ foi superior ao observado com a LCG Focus ${ }^{\circledR}$ 1-2 week $^{(16)}$. Outros autores ${ }^{(1,5)}$ encontraram menor indução de aberrações de alta ordem em LCG torneadas. Para alguns autores ${ }^{(1)}$ na comparação com as lentes tornea- 
das, as lentes moldadas e as centrifugadas produziram mais aberrações de alta ordem que degradaram a imagem retiniana e reduziram a sensibilidade ao contraste. Ambas as LCG empregadas neste estudo eram moldadas.

$\mathrm{O}$ indicador da imagem retiniana formado por um ponto de luz emitido por um objeto é denominado função de espalhamento do ponto ("point spread function", PSF). Esta medida fornece a informação da forma e da intensidade da distribuição da luz do ponto no plano da imagem. Com isto torna-se viável a avaliação da qualidade da imagem formada por este ponto. Para que a análise do PSF pudesse ser quantitativa adotou-se o índice numérico refletindo a relação entre o PSF de um sistema óptico em questão com o PSF ideal de um sistema óptico ideal (índice de Strehl). No estudo não houve diferença no índice de Strehl medido com óculos e com LCG e entre as LCG avaliadas (Figura 2). Outra variável analisada foi a função de transferência modular ("modulation transfer function"MTF). Trata-se de indicador que mede a capacidade do sistema óptico de transferir as informações do contraste do objeto para a imagem formada. Também, não houve diferença no MTF avaliado com e sem as LCG, e entre as LCG testadas (Figura 3).

\section{CONCLUSÃO}

Houve melhor desempenho óptico das lentes World Vision Disposable Asférica Wave Front ${ }^{\mathbb{R}}$ na medida de AV logMAR de alto contraste. Não houve diferença entre as LCG nas avaliações de sensibilidade ao contraste, índice de Strehl, MTF e de aberrações de alta ordem (aberrações totais, coma, trefoil, quadrifoil, aberração esférica e astigmatismo secundário).

\section{ABSTRACT}

Purpose: To evaluate the optical performance of eyes fitted with two different soft contact lenses: Acuvue ${ }^{\circledR} 2$ (Vistacon J\&J Vision Care Inc., USA) and World Vision Disposable Asférica Wave Front ${ }^{\circledR}$ (World Vision Ophthalmic, Brazil). Methods: An interventional prospective clinical trial studied a sample of 40 myopic patients $(-0.75 \mathrm{D}$ to $-4.50 \mathrm{D}$, with or without astigmatism up to $-0.75 \mathrm{D}$ ). Each patient had one eye randomized to visual performance evaluation including high and low contrast visual acuities, wavefront analysis and contrast sensitivity. Results: The Nidek OPD Scan detected a residual refraction (hypercorrection) for both contact lenses. High contrast visual acuity was significantly higher for World
Vision soft contact lenses. Low contrast visual acuity was similar for both soft contact lenses. Contrast sensitivity improved significantly at $1.5 ; 3 ; 6$ e 18 spatial frequencies for both soft contact lenses, but no difference was found between them. Regarding wavefront analysis, no difference was found between both soft contact lenses. There were not significantly differences in the Strehl indices and MTF for both soft contact lenses. Conclusion: World Vision Disposable Asférica Wave Front ${ }^{\circledR}$ soft contact lenses had a better performance regarding high contrast visual acuity. However, low contrast visual acuity, wavefront analysis and contrast sensitivity were similar for both soft contact lenses.

Keywords: Myopia; Eyeglasses; Contact lenses; Visual acuity; Contrast sensitivity; Prospective studies

\section{REFERÊNCIAS}

1. Jiang H, Wang D, Yang L, Xie P, He JC. A comparison of wavefront aberrations in eyes wearing different types of contact lenses. Optom Vis Sci. 2006; 83(10):769-74.

2. Lu F, Mao X, Qu J, Xu D, He JC. Monochromatic wavefront aberrations in the human eye with contact lenses. Optom Vis Sci. 2003;80(2):135-41.

3. Maldonanado-Codina C, Efron N. Impact of manufacturing technology and material composition on the clinical performance of hydrogel lenses. Optom Vis Sci. 2004;81(6):442-54.

4. Hill JF. Clinical comparison of the (polymacon) spin-cast hydrogel contact lens to the (polymacon) lathe-cut hydrogel lenses. Am J Optom Physiol Opt. 1980; 57(8):523-7.

5. Wachler BS, Phillips CL, Schanzilin DJ, Krueger RR. Comparison of contrast sensitivity in different contact lenses and spectacles. CLAO J. 1999;25(1):48-51.

6. Bailey IL. Visual acuity. In: Benjamin WJ, editor. Borish's clinical refraction. Philadelphia: WS Saunders; 1998. p.179-241.

7. Urbano AP, Leal M, Urbano I. Princípios básicos da acuidade visual. In: Urbano AP, editor. Wavefront. Cirurgia personalizada. Rio de Janeiro: Cultura Médica, 2006. p.59-65.

8. Wolkstein M, Atkin A, Bodis-Wolmer I. Contrast sensitivity in retinal disease. Ophthalmology. 1980;87(11):1140-9.

9. Sekuler R, Owsley C, Berenberger R. Contrast sensitivity during provoked visual impairment in multiple sclerosis. Ophtalmic Physiolol Opt. 1986;6: 229-32.

10. Grey CP. Changes in contrast sensitivity when wearing low, medium, and high water content soft lenses. J Br Contact Lens Assoc. 1986;9:21-5

11. Grey CP. Changes in contrast sensitivity during the first hour of soft contact lens wears. Am J Optom Physiol Opt. 1986;63(9):702-7.

12. Millodot M. Variation of visual acuity with contact lenses. A function of luminance. Arch Ophthalmol. 1969;82(4):461-5.

13. Millodot M. Variation of visual acuity with soft contact lenses: a function of luminance. Am J Optom Physiol Opt. 1965;52(8):541-4.

14. Cox I, Holden BA. Soft contact lens-induced longitudinal spherical aberration and its effect on contrast sensitivity. Optom Vis Sci. 1990;67(9):679-83.

15. Barreto J Jr, Netto MV, Cigna A, Bechara S, Kara-José N. Precision of higher order aberration repeatability with NIDEK OPD Scan retinoscopic aberrometry. J Refract Surg. 22(9 Suppl):S1037-40.

16. Barth B, Alves MR, Kara-José N. Desempenho visual na correção de miopia com óculos e lentes de contato gelatinosas. Arq Bras Oftalmol. 2008;71(1):90-6. 